\title{
Efeito do Perfil Granulométrico das Partículas Dietéticas sobre Parâmetros de Desem- penho de Vacas Leiteiras em Lactação
}

\author{
Edinaldo da Silva Bezerra1, Augusto César de Queiroz², Fabiana Maldonado3, \\ José Carlos Pereira², Mário Fonseca Paulino²
}

RESUMO - O objetivo deste experimento foi avaliar o efeito do perfil granulométrico das partículas dietéticas sobre o desempenho de vacas leiteiras em lactação. Dez vacas leiteiras da raça Holandesa (média de $550 \mathrm{~kg}$ ) foram distribuídas em um delineamento em switchback, em três períodos consecutivos. Os animais foram estabulados individualmente e receberam rações em mistura completa, constituída de feno de capim Tifton $(50,4 \%)$ e concentrado (49,6\%). O feno foi triturado em moinho comercial com peneiras de malhas de 3,$2 ; 4,8 ; 7,9 ; 15,9$; e 25,4 mm, e amostras de cada moagem foram submetidas ao separador de partículas modelo Penn State, para determinação do perfil de distribuição das partículas. Constituíram-se cinco tratamentos: T1 - 100\% das partículas abaixo de 8 mm; T2 - 76\% abaixo de $8 \mathrm{~mm}$ e $24 \%$ entre 8 e $19 \mathrm{~mm}$; T3 - 36,7\% abaixo de $8 \mathrm{~mm}, 26,6 \%$ entre 8 e $19 \mathrm{~mm}$ e $36,7 \%$ acima de $19 \mathrm{~mm}$; T4 - 32\% abaixo de $8 \mathrm{~mm}, 28 \%$ entre 8 e $19 \mathrm{~mm}$ e $40 \%$ acima de $19 \mathrm{~mm}$; e T5 - 26\% abaixo de $8 \mathrm{~mm}, 28 \%$ entre 8 e $19 \mathrm{~mm}$ e $46 \%$ acima de $19 \mathrm{~mm}$. Os resultados indicaram que os consumos de ração e nutrientes e a conversão alimentar não foram influenciados pelos diferentes perfis granulométricos das dietas, mas observaram-se variações na produção e na composição do leite. Verificou-se que vacas leiteiras alimentadas com dietas com perfis granulométricos intermediários produziram mais leite. A quantidade de gordura do leite produzida não foi afetada pelo perfil de distribuição das partículas dietéticas da dieta, porém vacas leiteiras alimentadas com dietas de maior granulometria produziram leite com maior teor de gordura. O processamento dos ingredientes das dietas com reduções do tamanho da partícula deve ser considerado criteriosamente, já que melhor desempenho ocorreu quando os animais foram submetidos às dietas de perfil granulométrico intermediário.

Palavras-chave: fibra, consumo alimentar, conversão alimentar, granulometria, desempenho

\section{Effect of the Physical Profile of the Dietary Particles on Intake and Production Parameters of Dairy Cows}

\begin{abstract}
The objective of this experiment was to evaluate the effects of different dietetic physical profile on the performance of lactating dairy cows. Ten Holstein dairy cows (avg. $550 \mathrm{~kg}$ ) were allotted to a switch back experimental design in three successive periods. The animals were individually housed in a tie stall and fed with total mixed rations constituted by Tifton hay (50,4\%) and concentrated (49,6\%). The Tifton hay was ground in commercial miller and sieved at 3,2;4,8; 7,9; 15,9 and 25,4 mm mesh and samples from each grinding were screened through the Particles Separator Model Penn State for the determination the profile of particle distribution. Five treatments were constituted: T1-100\% of particles below $8 \mathrm{~mm}$; T2-76\% below $8 \mathrm{~mm}$ and $24 \%$ between 8 and 19 $\mathrm{mm}$; T3-36,7\% below $8 \mathrm{~mm}, 26,6 \%$ between 8 and $19 \mathrm{~mm}$ and 36,7\% above $19 \mathrm{~mm}$; T4-32\% below $8 \mathrm{~mm}, 28 \%$ between 8 and $19 \mathrm{~mm}$ and $40 \%$ above $19 \mathrm{~mm}$; T5-26\% below $8 \mathrm{~mm}, 28 \%$ between 8 and $19 \mathrm{~mm}$ and $46 \%$ above $19 \mathrm{~mm}$. The results indicated that diet and nutrient intakes as well feed conversion were not affected by the different diet physical profile, but changes in the milk production and composition were observed. It was observed that dairy cows fed with an intermediary dietary distribution profile yielded more milk. Milk fat yielded was not affected by dietary fiber particle distribution profile however dairy cows fed with diets of higher physical profile yielded a higher milk fat content. The processing of the diet ingredients, with reduction in the particle size, should be carefully considered once the best performance response was observed when the animals were submitted to the diets with intermediary physical profile.
\end{abstract}

Key Words: fiber, feed intake, dry feed conversion, physical profile, performance

\section{Introdução}

Em alimentação de ruminantes, a ingestão de matéria seca é um dos aspectos mais importantes a serem considerados na formulação de dietas, por causa da sua estreita relação com o desempenho produtivo e reprodutivo. No caso de ruminantes, essas dietas ainda são baseadas em quantidades relativamente altas de forragens ricas em fibra em detergente neutro (FDN). Uma dieta com nível mais

\footnotetext{
1 Universidade Federal Rural-RJ, km 7 BR465, 23890 - 000, Seropédica - RJ. E.mail: naldobezerra@uol.com.br

2 Universidade Federal de Viçosa, Viçosa - MG., E.mail: aqueiroz@ufv.br; jearper@ufv.br; mpaulino@ufv.br

${ }^{3}$ Pós-graduação em Zootecnia, Universidade Federal de Viçosa, Viçosa - MG.
} 
alto de FDN pode garantir uma fermentação adequada, que proporcione aporte significativo de proteína e outros nutrientes de origem microbiana, e, conseqüentemente, reduzir custos com a alimentação; no entanto, ela também pode promover restrições ao consumo alimentar, tendo em vista a necessidade de maior tempo de permanência do alimento no rúmen, e limitar a exploração econômica em sistemas de produção mais intensivos, se a demanda nutricional for mais elevada. A relação entre a presença de FDN e a capacidade do animal em consumir alimentos tem sido investigada e, embora substanciais avanços tenham sido conseguidos, algumas questões ainda precisam ser elucidadas.

Argumenta-se que as diferenças de natureza química da fibra entre os ingredientes disponíveis para o balanceamento de dietas podem exercer influência na resposta animal e interferir na determinação das exigências em fibra. Contudo, a dificuldade prática de utilizar a composição química da fibra como um elemento de entrada na elaboração dos modelos de predição tem direcionado esforços no sentido de conseguir parâmetros quantitativos de fácil medição e que possam ser utilizados nesses modelos. Dessa forma, tem sido proposta a efetividade física como um parâmetro a ser utilizado na determinação da exigência de fibra pelo ruminante. A questão que se coloca, todavia, é até que ponto ela é uma boa estimativa das exigências de fibra pelo ruminante e como ela pode ser relacionada com o consumo de matéria seca e os padrões de fermentação ruminal.

As dietas para animais de alta produção são balanceadas com o objetivo de maximizar a ingestão de energia e a síntese microbiana, o que exige, em tese, alimentos altamente fermentáveis como fontes de energia para os microrganismos ruminais. Considerando que a fibra é menos fermentável que amido e açúcares, é de se esperar que o conteúdo em energia fermentável de uma dieta aumente à medida que se reduz a quantidade de fibra dessa dieta (Allen, 1996). Ocorre que, para se maximizar a produção bovina, além de uma densidade adequada de energia, os animais exigem também uma quantidade mínima de fibra para garantir ruminação e produção de saliva adequada (Sudweeks et al., 1981), digestão satisfatória da fibra (Mertens \& Loften, 1980) e manutenção do pH ruminal (Nocek, 1997).

O NRC (1989) sugere o atendimento mínimo de fibra a partir das quantidades de fibra em detergente neutro (FDN) e fibra em detergente ácido (FDA), porém esses parâmetros parecem não ser adequados (Van Soest, 1994; Santini et al., 1983), uma vez que diferenças no tamanho e na distribuição das partículas da fibra e o tempo de retenção podem variar (Allen, 1996), alterando sua eficiência no estímulo da mastigação e, conseqüentemente, na produção de saliva e manutenção do pH ruminal. Dessa forma, tem sido proposta a utilização de um coeficiente de efetividade da fibra, que poderia ser calculado com base nas respostas do animal diante das diferentes fontes de fibra presente nos alimentos.

A determinação do consumo de alimento pelo animal é fundamental para o balanceamento adequado de suas dietas, mas em ruminantes existem inúmeros fatores que interferem nesse consumo, com intensidade de diferentes magnitudes. A quantidade de alimento ingerido pode sofrer variações da ordem de 10 até $60 \%$, devido ao efeito de fatores inerentes ao ambiente, ao animal, ao alimento e às condições de manejo (Roseler et al., 1993).

A influência do alimento sobre o consumo de matéria seca está grandemente associada à sua composição em FDN, especialmente para animais em condições de alta produção que demandam quantidades elevadas de energia em suas dietas. A formulação de dietas objetivando conter adequados níveis de energia para atender altas produções normalmente resulta em rações com altos níveis de grãos em detrimento da fibra (Stokes, 1997), o que pode ocasionar problemas como acidose, laminite, cetose, deslocamento de abomaso, além de redução no teor de gordura do leite (Shearer, 1996).

Por outro lado, dietas com altos níveis de fibra impõem ao ruminante a necessidade de maior tempo de permanência do alimento e ampla capacidade ruminal em acomodar material de baixa densidade para que se processe uma fermentação adequada (NRC, 1989). Há possibilidade, nesses casos, de que o fill, ou enchimento ruminal, exerça efeito significativo sobre a capacidade do animal em consumir matéria seca. A distensão física do retículo-rúmen é o principal fator limitante na ingestão de muitas forragens e dietas ricas em fibra, mas também a presença de mecanorreceptores na parede ruminal é responsável por esse efeito (Forbes, 1995).

A necessidade de adequação da fibra da dieta para vacas em lactação já é conhecida por muitos produtores, tendo em vista que à ausência desse nutriente é atribuida série de distúrbios ruminais e metabólicos, que geram conseqüências para a saúde 
e o desempenho dos animais. Essa assertiva é corroborada por Allen (1996), para quem os ruminantes precisam de forragem em suas dietas para atender suas exigências em fibra e maximizar a produção e manter a saúde através da manutenção da estabilidade das condições ruminais. Entretanto, conforme observam Armentano e Pereira (1997), a definição dessas exigências é bastante complexa, porque o ajuste da fibra dietética com os ingredientes usuais da ração das vacas promove mudança em vários aspectos da dieta, que, por conseguinte, geram uma série de respostas inter-relacionadas no animal.

As diferenças nas quantidades e propriedades físicas da fibra podem afetar a utilização da dieta e, por conseqüentemente, o desempenho animal (Mertens, 1997). Quando muita fibra é colocada na ração, a densidade energética abaixa, a ingestão é reduzida e a produtividade diminui significativamente(Beauchemin, 1996; Mertens, 1996). Ao contrário, se a quantidade de fibra na dieta for muito reduzida, uma variedade de sintomas pode ocorrer, variando desde uma alteração no padrão de fermentação ruminal até uma acidose aguda, que pode levar à morte do animal (Mertens, 1996).

A ausência de efetividade da dieta é considerada por muitos autores (Grant et al, 1990a,b; Sudweeks et al., 1981; Woodford et al., 1986) como a principal causa de acidose subclínica e queda no teor de gordura do leite. A rápida fermentação observada em dietas com baixa fibra efetiva resulta em rápido acúmulo de ácidos no rúmen, reduzindo o $\mathrm{pH}$. Num efeito cascata, as bactérias acidificadoras acéticas que digerem fibra não sobrevivem ao abaixamento do $\mathrm{pH}$ e os microrganismos que produzem propionato e lactato se tornam preponderantes, causando mais acúmulo de ácidos e reduzindo ainda mais o $\mathrm{pH}$ ruminal. $\mathrm{O}$ acúmulo de ácidos promove danos ao epitélio ruminal, reduzindo a absorção de ácidos e colaborando também para o maior acúmulo (Ishler et al., 1996). A conseqüência é a diminuição na ingestão de matéria seca, seguida de queda na produção de leite e no teor de gordura.

Pequenas partículas alimentares são digeridas a taxas mais rápidas do que partículas maiores, porque apresentam maior área de superfície exposta por volume de tecido (Buxton \& Redfearn, 1997). No entanto, embora a trituração ou moagem das forragens forneça maior área de superfície para ação das enzimas, a utilização dos carboidratos estruturais não é aumentada (Varga \& Kolver, 1997). De fato, aumentos no desempenho animal são obtidos principalmente a partir de aumentos na ingestão de energia digestível (Bourquin et al., 1990).

Beauchemin (1996) verificou que pequenas diferenças no comprimento das partículas de silagem de alfafa foram capazes de afetar a produtividade de vacas leiteiras. Isso ocorreu, provavelmente, porque o aumento de 5 para $10 \mathrm{~mm}$ no comprimento das partículas favoreceu a atividade ruminal, especialmente no tempo de ruminação, quando se utilizou dieta contendo $12 \%$ de fibra em detergente neutro oriunda da forragem. Em dietas formuladas com $22 \%$ de FDN, não se observaram efeitos da mudança do comprimento da partícula da silagem, porque o aumento da FDN reduziu a ingestão de matéria seca.

Os trabalhos aqui desenvolvidos objetivaram avaliar o efeito de diferentes perfis granulométricos da fibra em dietas formuladas para vacas em lactação, com as mesmas proporções de volumoso e concentrado, sobre os seus desempenhos e sobre o consumo e a conversão de alimentos.

\section{Material e Métodos}

O trabalho com os animais foi desenvolvido no Setor de Bovinocultura de Leite do Departamento de Zootecnia (DZO) da Universidade Federal de Viçosa (UFV), na cidade de Viçosa - MG, entre os meses de março e julho de 1999. Os trabalhos de incubação em líquido de rúmen de sobras e alimentos foram realizados no Instituto de Zootecnia (IZ) da Universidade Federal Rural do Rio de Janeiro (UFRRJ). Análises químicas e bromatológicas foram realizadas nos Laboratórios de Nutrição Animal do DZO e do IZ e no Laboratório de Análise de Nitrogênio do Centro Nacional de Pesquisa em Agrobiologia (CNPAB) da EMBRAPA, Seropédica - RJ.

Foram utilizadas dez vacas com grau de sangue HPB variando entre $7 / 8$ e PC, entre a segunda e a quinta ordem de lactação, com peso vivo médio inicial variando de 450 a $650 \mathrm{~kg}$, todas elas já tendo atingido o pico de lactação. Foi utilizado o delineamento em switchback, conforme preconizado por Lucas (1960), com dez vacas distribuídas em dois blocos, utilizandose a ordem de parição como variável restritiva para composição dos blocos. As vacas foram alojadas em baias individuais do tipo tie stall, dotadas de comedouros e bebedouros individuais.

Os dados foram coletados por três períodos consecutivos de 28 dias, sendo 14 dias de adaptação dos animais às dietas e 14 dias de coleta de dados. Para compor os tratamentos, o feno foi submetido à desin-

R. Bras. Zootec., v.31, n.3, p.1511-1520, 2002 (suplemento) 
tegração com triturador comercial de facas, dotado de peneiras com malha de 3,$2 ; 4,8 ; 7,9 ; 15,9 ;$ e $25,4 \mathrm{~mm}$. Aproximadamente $100 \mathrm{~g}$ de amostra do material triturado em cada peneira foram colocados em separador de partículas modelo Penn State, de acordo com a metodologia proposta por Lammers et al. (1996) e em consonância com os procedimentos sugeridos pela ADSA (1970) para determinação do perfil granulométrico da dieta. Os tratamentos foram constituídos, então, de acordo com o perfil da fibra de cada material oriundo das peneiras de moagem, conforme ilustrado na Tabela 1.

Foram utilizadas dietas isonutricionais completas, constituídas por feno de tifton e suplemento concentrado elaborado com milho e soja, para todos os animais. As exigências nutricionais foram estabelecidas com base nas recomendações preconizadas pelo NRC (NRC, 1989).

Amostras dos ingredientes foram analisadas no Laboratório de Nutrição Animal da UFV, para determinação da composição nutricional (Tabela 2). As rações foram calculadas utilizando-se o pacote computacional Pudairy, desenvolvido pela Purdue University.

As dietas totais foram pesadas diariamente e oferecidas duas vezes ao dia, pela manhã e à tarde, imediatamente antes das ordenhas. A quantidade da mistura total foi ajustada no período de adaptação, a fim de garantir uma sobra de aproximadamente $10 \%$ do oferecido. As sobras foram retiradas antes do primeiro arraçoamento da manhã e pesadas para cálculo do consumo.

Amostras da ração e do feno foram coletadas e acondicionadas em sacos plásticos, devidamente identificadas e armazenadas em freezer para análises laboratoriais posteriores. As sobras foram retiraTabela 1 - Perfil de distribuição (\%) das partículas de
feno nos tratamentos

Table 1 - Hay particle profile distribution (\%) in the treatments

\begin{tabular}{|c|c|c|c|c|c|}
\hline \multirow[t]{2}{*}{$\begin{array}{l}\text { Tamanho da partícula } \\
\text { Particle size }\end{array}$} & \multicolumn{5}{|c|}{$\begin{array}{c}\text { Tratamento } \\
\text { Treatment }\end{array}$} \\
\hline & $\mathrm{T} 1$ & $\mathrm{~T} 2$ & $\mathrm{~T} 3$ & $\mathrm{~T} 4$ & T5 \\
\hline $\begin{array}{l}\text { Acima de } 19 \mathrm{~mm} \\
\text { Above } 19 \mathrm{~mm}\end{array}$ & 0,0 & 0,0 & 36,7 & 40,0 & 46,0 \\
\hline $\begin{array}{l}\text { Entre } 8 \text { e } 19 \mathrm{~mm} \\
\text { Between } 8 \text { and } 19 \mathrm{~mm}\end{array}$ & 0,0 & 24,0 & 26,6 & 28,0 & 28,0 \\
\hline $\begin{array}{l}\text { Acumulado }(\geq 8 \mathrm{~mm}) \\
\text { Above } 8 \mathrm{~mm}\end{array}$ & 0,0 & 24,0 & 63,3 & 68,0 & 74,0 \\
\hline $\begin{array}{l}\text { Abaixo de } 8 \mathrm{~mm} \\
\text { Below } 8 \mathrm{~mm}\end{array}$ & 100,0 & 76,0 & 36,7 & 32,0 & 26,0 \\
\hline $\begin{array}{l}\text { Acumulado total } \\
\text { Accumulate total }\end{array}$ & 100,0 & 100,0 & 100,0 & 100,0 & 100,0 \\
\hline
\end{tabular}

R. Bras. Zootec., v.31, n.3, p.1511-1520, 2002 (suplemento) das pela manhã e ao final do período de coleta, e as amostras foram retiradas, para análises posteriores.

Durante o período de avaliação da produção láctea, foram coletadas amostras de leite em quantidades proporcionais ao volume produzido nas ordenhas da manhã e da tarde. Amostras da manhã e da tarde foram misturadas proporcionalmente para formar uma amostra composta, a fim de serem analisadas em laboratório.

As amostras de sobras e de feno foram secadas em estufa ventilada a $65^{\circ} \mathrm{C}$ por 72 horas, para proceder à moagem. Ingredientes da ração e o material seco em estufa foram moídos em moinho tipo Willey dotado de peneira de $1,0 \mathrm{~mm}$, acondicionados em vidros com tampas e armazenados para posteriores análises laboratoriais.

Os materiais foram analisados em laboratório, para avaliação do conteúdo em matéria seca (MS), fibra em detergente ácido (FDA) e nitrogênio total (N) pelas metodologias descritas por Silva (1990). A fibra em detergente neutro (FDN), a lignina, o nitrogênio insolúvel em detergente neutro (NIDN) e o nitrogênio insolúvel em detergente ácido (NIDA) foram determinados pelas metodologias propostas por Van Soest et al. (1991). Para acessar a FDN indigestível, utilizou-se o protocolo adaptado de Pell \& Schofield (1993). Teores de cinzas e extrato etéreo bruto foram determinados pela metodologia do AOAC (1990). O teor em carboidratos não-fibrosos (CNF) foi estimado por diferença, usando-se a seguinte equação (Van Soest et al., 1991):

$$
\begin{aligned}
\mathrm{CNF}= & 100-[\mathrm{N} \text { total } \times 6,25+\text { Cinzas }+ \text { Extrato } \\
& \text { Etéreo }+(\text { FDN }-\mathrm{NIDN} \times 6,25)]
\end{aligned}
$$

Amostras de leite foram analisadas no Laboratório de Controle de Qualidade da Fundação Arthur Bernardes, para determinação de teor de gordura, teor de sólidos totais, densidade e acidez. A gordura foi determinada por fotometria. A acidez, a densidade e o teor de sólidos totais foram determinados segundo a recomendação do LANARA (1981).

Para analisar os dados de produção de leite, as médias foram comparadas utilizando-se a sugestão de Lucas (1956), em que os contrastes foram comparados como se segue:

$$
\mathrm{D}=\mathrm{Y}_{1}-2 \mathrm{Y}_{2}+\mathrm{Y}_{3},
$$

em que: $Y_{1}, Y_{2}$ e $Y_{3}$, as produções de cada animal no primeiro, segundo e terceiro períodos, respectivamente.

Os resultados para os demais parâmetros estudados foram analisados de acordo com o seguinte modelo estatístico: 
Tabela 2 - Composição bromatológica ${ }^{1}$ da ração total e de seus ingredientes

Table 2 - Nutritional composition of the ration and ingredients

\begin{tabular}{|c|c|c|c|c|c|c|c|}
\hline Item & $\begin{array}{c}\mathrm{MS}(\%) \\
D M\end{array}$ & $\begin{array}{c}\mathrm{PB}(\%) \\
C P\end{array}$ & $\begin{array}{l}\mathrm{FDN}(\%) \\
N D F\end{array}$ & $\begin{array}{c}\mathrm{FDA}(\%) \\
A D F\end{array}$ & $\begin{array}{c}\mathrm{EE}(\%) \\
E E\end{array}$ & $\begin{array}{l}\mathrm{MO}(\%) \\
O M\end{array}$ & $\begin{array}{l}\mathrm{CNF}(\%) \\
\text { NSC }\end{array}$ \\
\hline Milho moido & 87,93 & 7,46 & 13,97 & 1,42 & 4,15 & 98,35 & 55,72 \\
\hline $\begin{array}{l}\text { Corn, ground } \\
\text { Farelo de soja } \\
\text { Soybean meal }\end{array}$ & 85,56 & 42,62 & 9,35 & 7,82 & 1,95 & 94,61 & 40,69 \\
\hline $\begin{array}{l}\text { Calcário } \\
\text { Limestone }\end{array}$ & 100,00 & 0,00 & 0,00 & 0,00 & 0,00 & 0,00 & 0,00 \\
\hline $\begin{array}{l}\text { Fosf. bicálcico } \\
\text { Dicalcium phosphate }\end{array}$ & 100,00 & 0,00 & 0,00 & 0,00 & 0,00 & 0,00 & 0,00 \\
\hline $\begin{array}{l}\text { Sal } \\
\text { Salt }\end{array}$ & 100,00 & 0,00 & 0,00 & 0,00 & 0,00 & 0,00 & 0,00 \\
\hline $\begin{array}{l}\text { Supl. vitamínico } \\
\text { Premix vitamin }\end{array}$ & 100,00 & 0,00 & 0,00 & 0,00 & 0,00 & 0,00 & 0,00 \\
\hline $\begin{array}{l}\text { Supl. mineral } \\
\text { Premix mineral }\end{array}$ & 100,00 & 0,00 & 0,00 & 0,00 & 0,00 & 0,00 & 0,00 \\
\hline $\begin{array}{l}\text { Feno } \\
\text { Hay }\end{array}$ & 92,76 & 4,75 & 62,33 & 32,50 & 0,76 & 94,75 & 26,90 \\
\hline $\begin{array}{l}\text { Concentrado } \\
\text { Concentrate }\end{array}$ & 87,76 & 19,35 & 11,51 & 3,59 & 3,15 & 91,63 & 47,40 \\
\hline $\begin{array}{l}\text { Ração total } \\
\text { Total mix ration }\end{array}$ & 90,26 & 12,05 & 37,75 & 18,22 & 1,96 & 93,19 & 37,15 \\
\hline
\end{tabular}

${ }^{1}$ Expressa na base da matéria natural (Expressed in fresh matter basis).

$259 \%$ de milho moido (ground corn), $35 \%$ de farelo de soja (soybean meal), 1,9\% de calcário (limestone), 1,1\% de fosfato bicálcico (dicalcium phosphate), $1 \%$ de sal (salt), $1 \%$ de suplemento mineral (premix mineral) e $0,5 \%$ de suplemento vitamínico (premix vitamin).

$350,4 \%$ de feno de tifton, $49,6 \%$ de concentrado (50,4\% tifton hay, $49,6 \%$ concentrate).

$$
Y_{i j k l}=m+A_{i}+P_{j}+T_{k}+B_{1}+e_{i j k l}
$$

em que: $Y_{i j}$ o dado referente ao i-ésimo animal, no $j$ ésimo período, do k-ésimo tratamento; $\mathrm{m}$, a média geral observada; $\mathrm{A}_{\mathrm{i}} \mathrm{o}$ efeito do i-ésimo animal; $\mathrm{P}_{\mathrm{j}}, \mathrm{O}$ efeito do j-ésimo período; $\mathrm{T}_{\mathrm{k}}$, o efeito do k-ésimo tratamento; $\mathrm{B}_{1}$, o efeito l-ésimo bloco; e $\mathrm{e}_{\mathrm{ijk}}$, o erro aleatório associado ao i-ésimo animal, do j-ésimo período do k-ésimo tratamento. Os valores médios obtidos foram comparados pelo teste de Student Newman Keuls e a análise estatística foi realizada usando-se o SAEG - Sistema de Análises Estatísticas (UNIVERSIDADE FEDERAL DE VIÇOSA, 1996).

\section{Resultados e Discussão}

\section{Consumo e conversão alimentar}

$\mathrm{Na}$ Tabela 3 são apresentados os resultados de consumos de ração total e de matéria seca, assim como as médias de conversão alimentar. Os valores encontrados indicam que não houve efeito do perfil granulométrico das partículas alimentares das dietas sobre os padrões de consumo das rações e da matéria seca, nem sobre a eficiência de transformação dos alimentos em leite. Os resultados aqui reportados coincidiram com os observados por Kaske \&
Engelhardt (1990), que também não observaram efeito sobre o consumo de matéria seca e de nutrientes quando trabalharam com dietas em que se variou o perfil granulométrico.

Existem suficientes evidências na literatura para suportar o conceito de que a regulação de consumo por vacas leiteiras de alta produção depende de uma série de eventos, mas fundamentalmente da densidade energética e da capacidade de repleção (NRC, 1989; Roseler et al., 1993; Mertens, 1994; Van Soest, 1994; Hutjens, 1995).

Uma vez que as dietas eram isonutricionais, os níveis de energia e de FDN não poderiam ter influência sobre o consumo de matéria seca e, dessa forma, não poderiam ter reflexos sobre o consumo de nutrientes, indicando que esses resultados podem, de certa forma, ser considerados normais. Entretanto, os consumos de ração e de seus componentes nutricionais foram testados tendo em vista que o perfil da fibra da dieta poderia ter reflexos sobre o tempo médio de retenção da digesta e, assim, ter significativo efeito sobre o consumo de ração.

De acordo com Van Soest (1994), a ingestão de alimentos, especialmente os fibrosos, pode ser consideravelmente aumentada quando são processados 
Tabela 3 - Consumos absolutos (kg/cab/dia), relativos (\%/PV) e metabólicos (g/kg PV0,75) e conversão alimentar (kg de ração total/kg de leite produzido)

Table 3 - Absolute (kg/head/day), relative $(\% / L W)$ and metabolic feed intakes $\left(\mathrm{g} / \mathrm{kg} L W^{0,75}\right)$ and feed conversion (kg of TMR/ $\mathrm{kg}$ of milk)

\begin{tabular}{|c|c|c|c|c|c|c|}
\hline \multirow[t]{2}{*}{$\begin{array}{l}\text { Parâmetro } \\
\text { Parameter }\end{array}$} & \multicolumn{6}{|c|}{$\begin{array}{c}\text { Tratamento } \\
\text { Treatment }\end{array}$} \\
\hline & 1 & 2 & 3 & 4 & 5 & $\mathrm{CV}^{1}$ \\
\hline & \multicolumn{6}{|c|}{$\begin{array}{l}\text { Matéria natural } \\
\text { Fresh matter }\end{array}$} \\
\hline $\begin{array}{l}\mathrm{kg} / \mathrm{cab} / \mathrm{dia} \\
\mathrm{kg} / \mathrm{cow} / \mathrm{day}\end{array}$ & 21,52 & 22,95 & 22,49 & 20,95 & 22,78 & 12,35 \\
\hline $\begin{array}{l}\% / \mathrm{PV} \\
\% / L W\end{array}$ & 4,15 & 4,28 & 4,01 & 3,83 & 3,71 & 9,31 \\
\hline $\begin{array}{l}\mathrm{g} / \mathrm{kg} \mathrm{PV} V^{0,75} \\
\mathrm{~g} / \mathrm{kg} L W^{0,75}\end{array}$ & 198,10 & 206,00 & 194,70 & 185,10 & 184,40 & 8,99 \\
\hline & \multicolumn{6}{|c|}{$\begin{array}{l}\text { Matéria seca } \\
\text { Dry matter }\end{array}$} \\
\hline $\begin{array}{l}\mathrm{kg} / \mathrm{cab} / \mathrm{dia} \\
\mathrm{kg} / \text { cow/day }\end{array}$ & 19,42 & 20,72 & 20,30 & 18,91 & 20,56 & 12,35 \\
\hline $\begin{array}{l}\% / \mathrm{PV} \\
\% / L W\end{array}$ & 3,75 & 3,87 & 3,62 & 3,46 & 3,35 & 9,31 \\
\hline $\begin{array}{l}\mathrm{g} / \mathrm{kg} \mathrm{PV} 0,75 \\
g / k g L W^{0,75}\end{array}$ & 178,80 & 185,90 & 175,70 & 167,10 & 166,40 & 8,99 \\
\hline & & & $\begin{array}{r}\text { Conv } \\
\mathrm{Fe}\end{array}$ & $\begin{array}{l}\text { nentar } \\
\text { sion }\end{array}$ & & \\
\hline $\begin{array}{l}\mathrm{kg} \text { ração } / \mathrm{kg} \text { de leite } \\
\mathrm{kg} \text { of } T M R / \mathrm{kg} \text { of milk }\end{array}$ & 1,03 & 1,06 & 1,14 & 1,20 & 1,19 & 20,54 \\
\hline
\end{tabular}

${ }^{1}$ Coeficiente de variação $(\%)$.

1 Coefficient of variation (\%).

por moagem ou peletização, pois isso promove a desestruturação física da parede celular e o aumento da densidade da dieta, o que leva a maior taxa de passagem, menor tempo de ruminação e, concomitantemente, maior disponibilidade de tempo para consumir alimentos.

A densidade da partícula tem correlação negativa com o seu tamanho (Hungate, 1966; Sauvant, 1997), e as partículas com densidade inferior à unidade tendem a permanecer no rúmen por mais tempo (Kaske \& Engelhardt, 1990; Van Soest, 1994). Quando um bolo alimentar é formado, remastigado e redeglutido, as partículas mais finas são depositadas próximas ao orifício retículo omasal e aquelas mais pesadas passam mais facilmente pelo canal (Des Bordes \& Welch, 1984).

Os consumos relativos ao peso vivo dos animais variaram de 3,35 a 3,75\%, ficando dentro de uma faixa considerada normal para essa categoria animal, nas condições fisiológicas e de peso em que se encontravam, segundo os padrões de alimentação para gado leiteiro (Hoden et al., 1988; NRC, 1989; AFRC, 1993).

Na Tabela 4 encontram-se os dados de consumos médios diários de proteína bruta (PB), FDN, FDA, matéria orgânica (MO) e CNF, expressos em valores absolutos ( $\mathrm{kg} / \mathrm{cab} /$ dia) e em relação ao peso vivo metabólico $\left(\mathrm{g} / \mathrm{kg} \mathrm{PV}{ }^{0,75}\right)$.

Verifica-se que o perfil granulométrico das partículas dietéticas não teve efeito sobre o consumo de nutrientes pelos animais testados. Esses resultados certamente foram influenciados pelos mesmos padrões de consumo observados para a matéria seca e para a matéria orgânica. Tendência semelhante foi observada por Mooney \& Allen (1997) ao verificarem que o comprimento da partícula de silagem não influenciou a ingestão nem de matéria seca nem dos nutrientes por vacas holandesas em lactação. Grant et al. (1990a) também não verificaram efeito do tamanho da partícula de feno sobre as ingestões de matéria seca e dos nutrientes das rações por vacas leiteiras em início de lactação.

De fato, o consumo de matéria seca e de nutrientes de uma dieta para vacas leiteiras é muito dependente do status fisiológico da vaca (Campling, 1966; Hutjens, 1994) e da composição em FDN da dieta (Okine et al., 1997). Roseler et al. (1993) observaram que as altas produções de leite demandam grandes quantidades de nutrientes e que esse é um dos prin- 
Tabela 4 - Consumos de PB, FDN, FDA, MO e CNF

Table 4 - Intakes of CP, NDF, NDA, OM e NSC

\begin{tabular}{|c|c|c|c|c|c|c|}
\hline \multirow[t]{2}{*}{$\begin{array}{l}\text { Parâmetro } \\
\text { Parameter }\end{array}$} & \multicolumn{6}{|c|}{$\begin{array}{c}\text { Tratamento } \\
\text { Treatment }\end{array}$} \\
\hline & 1 & 2 & 3 & 4 & 5 & $\mathrm{CV}^{1}$ \\
\hline & & & & & & \\
\hline $\begin{array}{l}\mathrm{kg} / \mathrm{cab} / \mathrm{dia} \\
\mathrm{kg} / \mathrm{cow} \cdot \mathrm{day}\end{array}$ & 2,36 & 2,47 & 2,48 & 2,28 & 2,48 & 12,39 \\
\hline $\begin{array}{l}\mathrm{g} / \mathrm{kg} \mathrm{PV} \mathrm{PV}^{0,75} \\
\mathrm{~g} / \mathrm{kg} L W^{0,75}\end{array}$ & 20,10 & 20,20 & 21,40 & 22,20 & 21,70 & 9,79 \\
\hline & & & & & & \\
\hline $\begin{array}{l}\mathrm{kg} / \mathrm{cab} / \mathrm{dia} \\
\mathrm{kg} / \mathrm{cow} \cdot d a y\end{array}$ & 9,42 & 8,56 & 9,38 & 9,64 & 8,89 & 13,87 \\
\hline $\begin{array}{l}\mathrm{g} / \mathrm{kg} \mathrm{PV} 0,75 \\
g / k g L W^{0,75}\end{array}$ & 76,10 & 75,60 & 81,00 & 86,50 & 81,80 & 10,21 \\
\hline & & & & & & \\
\hline $\begin{array}{l}\mathrm{kg} / \mathrm{cab} / \mathrm{dia} \\
\mathrm{kg} / \mathrm{cow} \cdot d a y\end{array}$ & 4,05 & 3,77 & 4,09 & 4,20 & 3,84 & 14,27 \\
\hline $\begin{array}{l}\mathrm{g} / \mathrm{kg} \mathrm{PV} 0,75 \\
g / k g L W^{0,75}\end{array}$ & 32,80 & 33,30 & 35,30 & 37,60 & 35,40 & 10,55 \\
\hline & & & & & & \\
\hline $\begin{array}{l}\mathrm{kg} / \mathrm{cab} / \mathrm{dia} \\
\mathrm{kg} \text { cow.day }\end{array}$ & 21,42 & 19,82 & 21,21 & 21,67 & 20,39 & 12,29 \\
\hline $\begin{array}{l}\mathrm{g} / \mathrm{kg} \mathrm{PV} \mathrm{PV}^{0,75} \\
g / \mathrm{kg} L W^{0,75}\end{array}$ & 173,40 & 175,20 & 183,60 & 194,40 & 187,80 & 8,96 \\
\hline & & & & & & \\
\hline $\begin{array}{l}\mathrm{kg} / \mathrm{cab} / \mathrm{dia} \\
\mathrm{kg} / \text { cow.day }\end{array}$ & 9,11 & 8,45 & 9,02 & 9,17 & 8,84 & 11,27 \\
\hline $\begin{array}{l}\mathrm{g} / \mathrm{kg} \mathrm{PV} \mathrm{PV}^{0,75} \\
\mathrm{~g} / \mathrm{kg} L W^{0,75}\end{array}$ & 73,74 & 74,69 & 78,28 & 82,28 & 81,43 & 8,33 \\
\hline
\end{tabular}

cipais fatores responsáveis pelo estabelecimento do consumo de matéria seca.

Por outro lado, o imbalanço de nutrientes de uma dieta pode ser um fator inibidor do consumo de matéria seca e de nutrientes dessa dieta, devido ao aumento excessivo de metabólitos (Illius \& Jessop, 1996). Como as dietas eram isonutricionais e não se perceberam diferenças significativas nos consumos destas em função das mudanças de perfil granulométrico das rações, era esperado que também os nutrientes apresentassem a mesma tendência. Os consumos de nutrientes observados para todos os tratamentos foram suficientes para garantir um aporte nutricional adequado para sustentar a produção de leite e manter o escore corporal dos animais dentro da faixa desejada para o status fisiológico em que os animais se encontravam.

R. Bras. Zootec., v.31, n.3, p.1511-1520, 2002 (suplemento)

\section{Desempenho}

Na Tabela 5 encontram-se os dados médios de produção, composição e acidez do leite. Os resultados indicaram que houve efeito $(\mathrm{P}<0,05)$ do perfil granulométrico da fibra das dietas sobre o desempenho produtivo das vacas do experimento, notando-se que as aquelas alimentadas com dietas elaboradas para conter um perfil granulométrico intermediário da fibra apresentaram tendência a produzir mais leite e, em contraposição, as vacas alimentadas com dietas contendo partículas fibrosas mais grosseiras tenderam a alcançar menor produção.

O efeito da efetividade física da dieta sobre a produção de leite se dá de forma indireta, na medida em que podem exercer efeito sobre as quantidades consumidas, os padrões de consumo e a digestibilidade da matéria seca, bem como sobre os padrões de fermentação ruminal. 
Tabela 5 - Produção, concentração de sólidos e acidez do leite Table 5 - Milk yield, composition and acidity

\begin{tabular}{|c|c|c|c|c|c|c|}
\hline \multirow[t]{2}{*}{$\begin{array}{l}\text { Parâmetro } \\
\text { Parameter }\end{array}$} & \multicolumn{5}{|c|}{$\begin{array}{c}\text { Tratamento } \\
\text { Treatment }\end{array}$} & \multirow[b]{2}{*}{$\mathrm{CV}^{1}$} \\
\hline & 1 & 2 & 3 & 4 & 5 & \\
\hline $\begin{array}{l}\text { Leite (kg/cab/dia) } \\
\text { Milk (kg/cow.day) }\end{array}$ & $21,65^{\mathrm{ab}}$ & $24,02^{\mathrm{a}}$ & $22,49^{\mathrm{ab}}$ & $18,97^{\mathrm{b}}$ & $20,05^{a b}$ & 12,90 \\
\hline $\begin{array}{l}\text { Gordura }(\mathrm{g} / \mathrm{cab} / \mathrm{dia}) \\
\text { Fat }(\mathrm{g} / \text { cow.dav })\end{array}$ & 634,10 & 712,10 & 709,40 & 618,50 & 678,40 & 14,50 \\
\hline $\begin{array}{l}\text { Gordura }(\%) \\
\text { Fat }(\%)\end{array}$ & $3,39^{\mathrm{b}}$ & $3,57^{\mathrm{ab}}$ & $3,73^{\mathrm{ab}}$ & $3,98^{\mathrm{ab}}$ & $4,16^{\mathrm{a}}$ & 11,22 \\
\hline $\begin{array}{l}\text { Sólidos totais (g/cab/dia) } \\
\text { Total solids (g/cow.day) }\end{array}$ & 266,50 & 302,20 & 285,30 & 252,10 & 267,10 & 12,74 \\
\hline $\begin{array}{l}\text { Sólidos totais }(\%) \\
\text { Total solids }(\%)\end{array}$ & 12,29 & 12,64 & 12,74 & 13,30 & 13,34 & 5,35 \\
\hline $\begin{array}{l}\text { Acidez }\left({ }^{\circ} \text { Dornic }\right) \\
\left.\text { Acidity ( }{ }^{\circ} \text { Dornic }\right)\end{array}$ & 17,13 & 16,69 & 16,19 & 17,78 & 16,01 & 6,68 \\
\hline
\end{tabular}

${ }_{1}^{1}$ Médias na linha, seguidas de letras distintas, diferem entre si $(P<0,05)$.

${ }^{1}$ Means within a row followed by different letters differ $(P<.05)$.

Armentano \& Pereira (1997), revisando na literatura inúmeros trabalhos publicados relativos ao estudo da efetividade da fibra, verificaram correlação significativa entre a produção de leite, o tempo de mastigação e a concentração de fibra das forragens, fato também observado por Dado \& Allen (1994). Essa relação, todavia, nem sempre é bem estabelecida, pois, em geral, um dos propósitos de se acessar a fibra efetiva de uma dieta é permitir que ela possa ser elaborada com o máximo possível de carboidratos de digestão facilitada, na tentativa de maximizar a produção de leite, ao mesmo tempo, garantir a manutenção da saúde do animal e do teor de gordura do leite (Van Horn, 1997). Dessa forma, normalmente são feitas alterações nos níveis ou na qualidade da fibra a fim de garantir a melhor relação entre a síntese microbiana e o escape ruminal de nutrientes de fácil assimilação pelo hospedeiro, o que, de acordo com Mertens (1996, 2000), confundiria os efeitos físicos e químicos da fibra dietética.

Diferenças na quantidade e nas propriedades físicas da fibra podem afetar a utilização da dieta e o desempenho do animal, segundo Mertens (1997), principalmente em razão do efeito sobre o consumo e sobre as alterações na fermentação ruminal. Mooney \& Allen (1997), todavia, não observaram efeito do tamanho da partícula da silagem de alfafa sobre o desempenho leiteiro de vacas holandesas, resultado semelhante ao do trabalho de Swain \& Armentano (1994).

As diferenças notadas para a produção de gordura não foram significativas, em que pesem os valores absolutos terem se apresentado discrepantes. Um alto coeficiente de variação observado para esse parâmetro pode explicar esse resultado estatístico. É importante salientar que o efeito da efetividade da fibra dietética é preponderante sobre o percentual de gordura do leite, porém é realmente pouco provável que esse efeito persista sobre a produção absoluta de gordura, tendo em vista que o teor de gordura guarda também correlação intrínseca e negativa com o total de leite produzido, ambos com forte componente genético determinando sua expressão.

As dietas tiveram influência $(\mathrm{P}<0,05)$ no teor de gordura do leite dos animais em teste, com aumento progressivo e significativo da concentração láctea à medida que se aumentou a efetividade física da dieta. Esses resultados são consistentes com a tese de que o tamanho da partícula dietética exerce efeito marcante sobre o teor de gordura do leite de vacas de alto desempenho (O’Dell et al., 1968; Varga, 1997). Alteração na concentração de propionato no pool ruminal possivelmente pode ser um dos principais responsáveis por esse resultado (Grant et al., 1990a). Dietas com baixa efetividade física diminuem a atividade de ruminação e o pH ruminal (Welch, 1982), aumentam a concentração ruminal de propionato e podem desencadear a queda na concentração de gordura do leite mediante mudanças nas concentrações séricas de insulina (Grant et al., 1990a,b).

O aumento da concentração sérica de insulina pode promover aumento na concentração de glicose, na concentração de propionato ou na concentração de ambos, resultando na redução da mobilização de 
gordura corporal (Jenny et al., 1972; Jenny \& Polan, 1975) e, conseqüentemente, reduzindo a disponibilidade dos precursores de gordura do leite para a glândula mamária. Além disso, segundo Benson et al. (1972), a insulina pode aumentar a absorção de acetato pelos adipócitos e melhorar a atividade da lipase lipoprotéica, favorecendo, assim, a lipogênese no tecido adiposo.

A produção e o teor de sólidos totais e a acidez do leite do leite não foram influenciados pela efetividade das dietas. A produção e a concentração de sólidos totais são grandemente dependentes de fatores genéticos e guardam correlação estreita com o volume de leite produzido em cada lactação, podendo ser influenciados por raça, estágio de lactação e estação do ano (Homan \& Wattiaux, 1996). Portanto, é pequena a influência de fatores nutricionais, embora algumas características químicas da dieta possam ter algum efeito. A acidez pode ser influenciada pela dieta, mas os fatores de manejo, especialmente os de ordem higiênica e profilática, podem ser preponderantes em influenciar a acidez.

\section{Conclusões}

O consumo alimentar não foi afetado pelo perfil granulométrico das partículas dietéticas, sugerindo que talvez outros fatores poderiam ser os principais responsáveis na determinação da ingestão de alimentos.

Os resultados sugerem que o processamento dos ingredientes das dietas com reduções no tamanho da partícula devem ser considerados criteriosamente, já que, de modo geral, as respostas mais positivas, do ponto de vista do desempenho, ocorreram quando os animais foram submetidos às dietas de efetividade física intermediária.

\section{Literatura Citada}

AGRICULTURAL AND FOOD RESEARCH COUNCIL AFRC. Energy and protein requirements of ruminants. Wallingford: CAB International, 1993. 159p.

ALLEN, M.S. Fiber requirements for dairy cattle: how low can you go? 1996. http:// dairyext.tamu.edu/EXT_DATA/ fiber_low.htm. (06/02/98).

AMERICAN DAIRY SCIENCE ASSOCIATION - ADSA. A report: committee on classification of particle size in feedstuffs. Journal of Dairy Science, v.53, n.5, p.689-690, 1970.

ARMENTANO, L.E., PEREIRA, M. Measuring the effectiveness of fiber by animal response trials. Journal of Dairy Science, v.80, n.7, p.1416-1425, 1997.

ASSOCIATION OF OFFICIAL ANALYTICAL CHEMISTS AOAC. Official methods of analysis. 15.ed., Arlington. 1990. 1117 p.

R. Bras. Zootec., v.31, n.3, p.1511-1520, 2002 (suplemento)
BEAUCHEMIN, K.A. Using ADF and NDF in dairy cattle diet formulation - a western Canadian perspective. Animal Feed Science and Technology, v.58, n.1, p.101-111, 1996.

BENSON, J.D.; ASKEW, E.W.; EMERY, R.S. et al. Metabolism of fatty acids by adipose tissue and liver of cows fed normal, restricted roughage, or $\mathrm{MgO}$ supplemented rations. Journal of Dairy Science, v.55, n.1, p.83, 1972.

BOURQUIN, L.D.; GARLEB, K.A.; MERCHEN, N.R. et al. Effects of intake and forage level on site and extent of digestion of plant cell wall monomeric components by sheep. Journal of Animal Science, v.68, n. 12, p. 2479-2495, 1990.

BUXTON, D.R.; REDFEARN, D.D. Plant limitations to fiber digestion and utilization. Journal of Nutrition, v.127(suppl.), p.814-818, 1997.

CAMPLING, R.C. The intake of hay and silage by cows. Journal British Grassland Society, v.21, n.1, p.41-48, 1966.

DADO, R.G.; ALLEN, M.S. Intake limitations, feeding behavior, and rumen function of cows challenged with rumen fill from dietary fiber on inert bulk. Journal of Dairy Science, v.77, n.1, p.132-144, 1994.

DES BORDES, C.K.; WELCH, J.G. Influence of specific gravity on rumination and passage of indigestible particles Journal of Animal Science, v.59, n.2, p.470-475, 1984.

FORBES, J.M. Physical limitation of feed intake in ruminants and its interaction with other factors affecting intake. In: ENGELHARDT, W.W.; LEONHARD-MAREK, S.; BREVES, G. et al. (Eds.). Ruminant physiology: digestion, metabolism, growth and reproduction. Stuttgart: Enke, 1995. p.217-232.

GRANT, R.J.; COLENBRANDER, V.F.; MERTENS, D.R. Milk fat depression in dairy cows: role of particle size of alfalfa hay. Journal of Dairy Science, v.73, n.12, p.1823, 1990a.

GRANT, R.J.; COLENBRANDER, V.F.; MERTENS, D.R. Milk fat depression in dairy cows: role of silage particle size. Journal of Dairy Science, v.73, n.12, p.1834, 1990b.

HODEN, A.; COULON, J.B.; FAVERDIN, P. Alimentação de vacas leiteiras. In: JARRIGE, R. (Ed.) Alimentação de bovinos, ovinos e caprinos. Paris: INRA, 1988. p.132-154.

HOMAN, E.J.; WATTIAUX, M.A. Technical dairy guide: lactation and milking. 2.ed. Madison: University of Wisconsin, 1996. 94p.

HUNGATE, R.E. The rumen and its microbes. New York: Academic Press, 1966. 533p.

HUTJENS, M.F. Considerações sobre a alimentação de vacas leiteiras de alta produção. In: SIMPÓSIO INTERNACIONAL SOBRE PRODUÇÃO INTENSIVA DE LEITE, 1994, São Paulo. Anais... São Paulo: Universidade de São Paulo, 1994, p.1-9.

HUTJENS, M.F. Feeding applications for the high producing cow. In: CORNELL NUTRITION CONFERENCE FOR FEED MANUFACTURERS, 1995, Rochester. Proceedings... Ithaca: Cornell University, 1995. p.34-41.

ILLIUS, A.W.; JESSOP, N.S. Metabolic constraints on voluntary intake in ruminants. Journal of Animal Science, v.74, n.14, p.3052-3062, 1996.

ISHLER, V.; HEINRICHS, J.; VARGA, G. From feed to milk: understanding rumen function. Willard Building: Penn State Cooperative Extension, 1996. 52p. (Extension Circular, 422).

JENNY, B.F.; POLAN, C.E. Postprandial blood glucose and insulin in cows fed high grain. Journal of Dairy Science, v.58, n.2, p.512, 1975.

JENNY, B.F.; POLAN, C.E.; CHANDLER, P.T. Acetate and stearate utilization by lactating cows fed high grain restricted 
roughage rations. Journal of Dairy Science, v.55, n.5, p.1481, 1972.

KASKE, M.; Von ENGELHARDT, W. The effect of size and density on mean retention time of particles in the gastrointestinal tract of sheep. British Journal of Nutrition, v.63, p.683, 1990.

LAMMERS, B.P.; BUCKMASTER, D.R.; HEINRICHS, A.J. A simple method for the analysis of particle sizes of forage and total mixed rations. Journal of Dairy Science, v.79, n.5, p.922-928, 1996.

LANARA - Laboratório Nacional de Referência Animal. Métodos analíticos oficiais para controle de produtos de origem animal e seus ingredientes. II. Métodos físicos e químicos. Brasília, Ministério da Agricultura, 1981. (não paginado)

LOGGINS, P.E.; AMMERMAN, C.B.; MOORE, J.E. et al. Effect of feeding long hay or sodium bicarbonate with ground or pelleted diets high in citrus pulp on lamb performance. Journal of Animal Science, v.42, n.3, p.745, 1964.

LUCAS, H.L. Switch-back trials for more than two treatments. Journal of Dairy Science, v.39, n.2, p.146-154, 1956.

LUCAS, H.L. Critical features of good dairy feeding experiments. Journal of Dairy Science, v.43, n.3, p.193-212, 1960.

MERTENS, D.R. Regulation of forage intake. In: FAHEY Jr., G. (Ed.). Forage quality, evaluation, and utilization. Madison: American Society of Agronomy, 1994. p.450-493.

MERTENS, D. Using fiber and carbohydrate analyses to formulate dairy rations. In: INFORMATIONAL CONFERENCE WITH DAIRY AND FORAGE INDUSTRIES, 1996, Virginia. Proceedings... Virgínia: US Dairy Forage Research Center, 1996. p.81-92.

MERTENS, D.R. Creating a system for meeting the fiber requirements of dairy cows. Journal of Dairy Science, v.80, n.7, p.1463-1481, 1997.

MERTENS, D.R. Physically effective NDF and its use ind dairy rations explored. Feedstuffs, v.10, n.4, p.16-20, 2000.

MERTENS, D.R.; LOFTEN, J.R. The effects of starch on forage fiber digestion kinetics in vitro. Journal of Dairy Science, v.63, n.5, p.1437, 1980.

MOONEY, C.S.; ALLEN, M.S. Physical effectiveness of the neutral detergent fiber of whole linted cottonseed relative to that of alfalfa silage at two lengths of cut. Journal of Dairy Science, v.80, n.9, p.2052-2061, 1997.

NOCEK, J.E. Feeding management of the postpartum cow. In: SIMPÓSIO INTERNACIONAL DE DIGESTIBILIDADE EM RUMINANTES, 1997, Lavras. Anais... Lavras: Universidade Federal de Lavras, 1997. p.69-85.

NATIONAL RESEARCH COUNCIL - NRC. Nutrient requirements of domestic animals. Nutrient requirements of dairy cattle. 6 .ed revisada. Washington, D.C.: National Academy of Science, 1989.157p.

O'DELL, G.D.; KING, W.A.; COOK, W.C. Effect of grinding, pelleting, and frequency of feeding alfalfa hay or concentrate ad libitum as the sole feed for milking cows. Journal of Dairy Science, v.51, n.1, p.50, 1968.

OKINE, E.K.; KHORASANI, G.R.; KENNELY, J.J. Effects of source of forage and level of concentrate on chewing activity and milk production response in late lactation cows. Canadian Journal of Animal Science, v.77, n.2, p.253258, 1997.

PELL, A.N.; SCHOFIELD, P. Computerized monitoring of gas production to measure forage digestion in vitro. Journal of Dairy Science, v.76, n.4, p.1063-1073, 1993.
ROSELER, D.K.; FOX, D.G.; CHASE, L.E. et al. Feed intake prediction and diagnosis in dairy cows. In: CORNELL NUTRITION CONFERENCE FOR FEED MANUFACTURERS, 1993, Rochester. Proceedings... Ithaca: Cornell University, 1993. p.216-226.

SANTINI, F.J.; HARDIE, A.R.; JORGENSEN, N.A. Proposed use of adjusted intake based on forage particle length for calculation of roughage indexes. Journal of Dairy Science, v.66, p.811, 1983.

SAUVANT, D. Rumen mathematical modelling. In: HOBSON, P.N.; STEWART, C.S. (Eds.) The rumen microbial ecosystem. 2.ed. London: Blackie Academic \& Professional, 1997. p.685-708.

SHEARER, J.K. Lameness in dairy cattle: laminitis, claw disease, digital dermatitis, and foot root. Journal of Dairy Science, v.79(supp1.), p.189, 1996.

SILVA, D.J. Análise de alimentos (Métodos químicos e biológicos). Viçosa, MG: Universidade Federal de Viçosa, 1990. 165p.

STOKES, S.R. Particle size and ration uniformity: Is it important to the cow? In: WESTERN CANADIAN DAIRY SEMINAR, 1997, Alberta. Proceedings... Stephenville: Texas A\&M University, 1997. p.1-10.

SUDWEEKS, E.M.; ELY, L.O.; MERTENS, D.R. et al. Assessing minimum amounts and form roughages in ruminal diets: roughages value index system. Journal of Animal Science, v.53, n.6, p.1406, 1981.

SWAIN, S.M.; ARMENTANO, L.E. Quantitative evaluation of fiber from nonforage sources used to replace alfalfa silage. Journal of Dairy Science, v.77, n.8, p.2318-2331, 1994.

UNIVERSIDADE FEDERAL DE VIÇOSA - UFV. SAEG Sistema de Análise Estatística e Genética. Versão 7.1. Viçosa, 1996. 150p. (Manual do usuário).

VAN HORN, H.H. Maximizing milk production or minimizing cost with the use of byproduct feedstuffs. In: ANNUAL FLORIDA RUMINANT NUTRITION SYMPOSIUM, 8., Gainesville. Proceedings... Gainesville, Florida: University of Florida, p.23-36, 1997.

Van SOEST, P.J. Nutritional ecology of the ruminant. 2.ed., Ithaca: Comstock, 1994. 476p.

Van SOEST, P.J.; ROBERTSON, J.B.; LEWIS, B.A. Methods for dietary fiber, neutral detergent fiber, and nonstarch polysaccharides in relation to animal nutrition. Journal of Dairy Science, v.74, n.10, p.3583-3597, 1991.

VARGA, G.A. Fiber in the ration: how effective should it be? In: CORNELL NUTRITION CONFERENCE FOR FEED MANUFACTURERS, 59., 1997, Rochester. Proceedings... Ithaca: Cornell University, p.117-126, 1997.

VARGA, G.A.; KOLVER, E.S. Microbial and limitations to fiber digestion and utilization. Journal of Nutrition, v.127(suppl.), p.819-823, 1997.

WELCH, J.G. Rumination, particle size and passage from the rumen. Journal of Animal Science, v.54, n.6, p.1582, 1982.

WOODFORD, J.A.; JORGENSEN, N.A.; BARRINGTON, G.P. Impact of dietary fiber and physical form on performance of lactating dairy cows. Journal of Dairy Science, v.69, n.5, p.1035, 1986. 